\title{
Aspects épidémiologiques du diabète de type 1 à la banque d'insuline de Cotonou (Bénin)
}

\author{
Kaossarath A. FAGBEMI ${ }^{1 *}$, Simon AZONBAKIN ${ }^{1}$, Marius ADJAGBA ${ }^{1}$, \\ Lamine BABA-MOUSSA ${ }^{2}$ et Anatole LALEYE ${ }^{1}$ \\ ${ }^{I}$ UFR de Biologie Humaine, Faculté des Sciences de la Santé, Université d'Abomey-Calavi, Bénin. \\ ${ }^{2}$ Laboratoire de Biologie et de Typage Moléculaire en Microbiologie, Université d'Abomey-Calavi, Bénin. \\ *Auteur de correspondant ; E-mail: komsarath@yahoo.fr ; Tél. :(+229) 97-05-43-09.
}

\section{REMERCIEMENTS}

Cette étude a été réalisée grâce aux soutiens financiers du Ministère de l'Enseignement Supérieur et de la Recherche Scientifique (MESRS) et de l'Institut des Sciences Biomédicales Appliquées (ISBA) du Bénin.

\section{RESUME}

Le diabète sucré, bien qu'étant considéré comme une maladie des pays riches, constitue de plus en plus une préoccupation majeure dans les pays en développement et particulièrement en Afrique subsaharienne. L'objectif de cette étude était de déterminer les caractéristiques sociodémographiques, d'évaluer la fréquence du diabète de type 1 (DT1) et de calculer le taux de mortalité chez les diabétiques de type 1 ayant consultés à la Banque d'Insuline de Cotonou de janvier 1996 à décembre 2015. Une recherche bibliographique initiale a été menée début septembre 2012 et mise à jour systématiquement jusqu'à fin mars 2017. Des fiches d'enquêtes ont été préétablis pour recueillir dans un premier temps des informations contenues dans les dossiers des patients DT1 en général et dans un second temps des informations relatives aux patients DT1 qui ont pu être contactés. Au total, 12000 dossiers ont été étudiés dont 103 dossiers de patients DT1. La fréquence du DT1 retrouvée est de $0,9 \%$. Un taux de mortalité de $34 \%$ a été relevé dans le groupe des patients diabétiques de type 1 avec une part de mortalité de $54,3 \%$ chez les hommes et $45,7 \%$ chez les femmes.

(C) 2017 International Formulae Group. All rights reserved.

Mots clés : Caractéristiques sociodémographiques, fréquence, mortalité, diabète de type 1, Bénin.

\section{Epidemiological aspects of type 1 diabetes in the Insulin center of Cotonou (Benin)}

\begin{abstract}
The diabetes mellitus, long time considered as a disease of the developed countries, is more and more becoming a major concern in developing countries and particularly in sub-Saharan Africa. The aim of this study was to determine the sociodemographic characteristics, to estimate the frequency of the type 1 diabetes (T1D) among all type of diabetes and to calculate the mortality rate in the patients with T1D registered at the "insulin center" of Cotonou from January 1996 to December 2015. Firstly, a bibliographical research was effectuated from September, 2012 and regularly updated until March 2017. Investigations sheets were pre-
\end{abstract}


established firstly to collect data contained in the files of all the T1D patients, and secondly, data concerning the only T1D patients who are contacted during the study. At all, 12000 patients' files were studied and 103 files of patients suffering from T1D. The frequency of the T1D at the "insulin center" of Cotonou was $0.9 \%$. The mortality rate was $34 \%$ in the group of the T1D patients with $54.3 \%$ belonging to men and $45.7 \%$ to the women.

(C) 2017 International Formulae Group. All rights reserved.

Keywords: Socio-demographic characteristics, frequency, mortality, type 1 diabetes, Benin.

\section{INTRODUCTION}

Le diabète est généré par des désordres métaboliques chroniques caractérisés par une glycémie à jeun supérieure ou égale à $1.26 \mathrm{~g} / \mathrm{l}$. L'hyperglycémie est due à un déficit relatif ou absolu de la sécrétion et/ou de l'action de l'insuline (American Diabetes Association, 2010). Ce n'est donc pas une maladie unique mais plutôt un ensemble d'anomalies métaboliques et pathologiques, avec une variété de causes environnementales et héréditaires (Jansse et al., 2007). L’âge, le sexe, la profession, le revenu et l'antécédent familial représenteraient les facteurs de risques d'apparition du diabète (Koevi et al., 2014). Le diabète est l'une des maladies non transmissibles les plus répandues dans le monde, avec un effectif de patients diabétiques en 2014 qui avoisinait 422 millions de personnes (OMS, 2016). Selon les estimations faites en 2010 pour l'horizon 2030, cet effectif s'élèverait entre 4,4 et $4,7 \%$ de la population mondiale (Shaw et al., 2010). Parmi les diabétiques, environ $10 \%$ seraient atteints du DT1, 85\% du DT2 et les $5 \%$ restant concerneraient les autres types de diabète (American Diabetes Association, 2014). Sur les 347 millions de personnes atteintes du diabète dans le monde, 10 à 15 millions font le diabète de type 1 donc c'est le diabète de type 2 qui est la forme prédominante et ceci même en Afrique subsaharienne (Hall et al., 2011).

Le diabète de type 1 survient le plus souvent chez les enfants, les adolescents et les jeunes adultes. Ce type de diabète, autrefois appelé diabète insulinodépendant ou insulinovital, est dû à la destruction des cellules du pancréas spécialisées dans la production d'insuline : les cellules bêta des îlots de Langerhans. Cette destruction est elle-même causée par une réaction auto-immune, comme en témoigne la présence d'autoanticorps (Notking et Lernmark, 2001).

Au Bénin, les études montrent que la prévalence du diabète de type 2 s'est accrue ces dernières années. Ainsi en 2010, cette prévalence était estimée à 3\% (Mbanya et al., 2010) puis, 2 ans plus tard, elle avoisinait 4,2\% (Djrolo et al., 2012). Cependant, il n'existe aucune information relative à la prévalence du diabète de type 1 au Bénin. Spécifiquement, il s'est agi, dans cette étude, de décrire les caractéristiques sociodémographiques des diabétiques de type 1 , de déterminer la fréquence du diabète de type 1 dans l'ensemble des diabètes tous types confondus à la Banque d'Insuline de Cotonou au cours de ces vingt dernières années et de calculer le taux de mortalité au sein des patients diabétiques de type 1 . 


\section{MATERIEL ET METHODES}

\section{Echantillons}

Il s'agit d'une étude rétrospective qui a pris en compte tous les dossiers des patients diabétiques tous types confondus venant en consultation à la Banque d'Insuline de Cotonou au cours de la période allant de janvier 1996 à décembre 2015. La banque d'insuline de Cotonou est un centre de référence de diagnostic et de prise en charge des diabétiques venant d'un peu partout du territoire national du Bénin. Ont été éligibles pour cette étude, les patients diabétiques de type 1 d'origine béninoise, diagnostiqués avant l'âge de 30 ans par un endocrinologue suivant les recommandations de l'American Diabetes Association (ADA) (American Diabetes Association, 2014) et ayant donné leur consentement éclairé.

\section{Méthodes}

Les dossiers des patients diabétiques ont été étudiés et répartis selon le type de diabète. Deux fiches d'enquête ont été préétablies : la première a servi à recueillir les informations contenues dans les dossiers des patients diagnostiqués diabétiques de type 1 ; la seconde a été conçue pour les diabétiques de type 1 qui ont pu être contactés. Les fiches renseignent sur l'identité, l'âge, le sexe, l'ethnie, la profession, les complications et les antécédents familiaux liés au type de diabète. La part des patients DT1 au sein des diabétiques et le taux de mortalité ont été calculés à partir des formules standards.

\section{Analyses statistiques}

Les données recueillies au cours de cette étude ont été colligées à l'aide du tableur MS Excel 2013. La part des patients diabétiques de type 1 au sein des diabétiques et le taux de mortalité ont été calculés à partir des formules standards :

Fréquence $=$ Nombre de cas de diabète de type 1 enregistrés / Nombre de cas de diabète tout type confondu;

Taux de mortalité $=$ Nombre de décès enregistrés chez les patients diabétiques de type 1 / Nombre de patients diabétiques de type 1 .

Les analyses ont été effectuées grâce au logiciel SAS (version 9.1 SAS Institute Inc., Cary, NC, USA).

\section{RESULTATS}

La Figure 1 présente visuellement le flux des données issues du dépouillement des dossiers des patients diabétiques à la banque d'insuline. Au total, 12000 dossiers ont été étudiés. Des cas d'intolérance au glucose et de diabète gestationnel ont été également recensés dans l'étude. Sur les 103 patients DT1 recensés, seulement 52 ont pu être contactés contre 35 décès, 07 dossiers incomplets et 09 perdus de vue.

Cent trois patients diabétiques de type 1 ont été recensés au cours de ces 20 dernières années. Dans cet échantillon qui comprend $48,6 \%$ d'hommes et $51,4 \%$ de femmes, la tranche d'âge la plus représentée est celle de [8-16 ans [avec un pourcentage de 69,9\%. Les élèves et les patients d'ethnie Fon sont les plus impliqués, avec des pourcentages respectifs de $59,2 \%$ et $41,3 \%$. Dans $41,6 \%$ des cas, il existe des antécédents familiaux liés au diabète en général: $40,6 \%$ pour le type 2 et $1 \%$ pour le type 1 (Tableau 1 ). 
$\mathrm{Au}$ total, 51 patients diabétiques de type 1 ont pu être contactés parmi lesquels $54,9 \%$ sont de sexe féminin et $45,1 \%$ de sexe masculin. La tranche d'âge la plus représentée est celle de [8-16 ans [avec un pourcentage de $76,4 \%$. Les élèves et les patients d'ethnie Fon sont les plus impliqués avec des pourcentages respectifs de $60,8 \%$ et $52,9 \%$. Dans $56,9 \%$ des cas, il existe des antécédents familiaux liés au diabète en général: $54,2 \%$ pour le type 2 et $2 \%$ pour le type 1 (Tableau 2 ).

Les complications à type de macro et micro-angiopathies ont été recensées dans l'étude. $15,7 \%$ souffrent de complication du DT1 à type d'HTA. Un dysfonctionnement érectile a été observé chez $34,4 \%$ des sujets masculins. Une irrégularité, voire une absence de menstrues ont été observées respectivement chez $53,6 \%$ et $3,6 \%$ des sujets de sexe féminin. Aussi, 41,2\% de l'ensemble des patients ont une mauvaise acuité visuelle (Tableau 3).

Le diabète de type 2 occupe une place prépondérante avec une fréquence de $98,8 \%$, le diabète de type $1,0,9 \%$ et les diabètes secondaires $0,04 \%$. Des cas de diabètes secondaires liés par exemple à une pancréatite chronique calcifiante (PCC), ou une malnutrition n'ont pas été retrouvés dans cette étude (Tableau 4). Un taux de mortalité de $34 \%$ a été recensé avec une part de mortalité de $54,3 \%$ chez les hommes et $45,7 \%$ chez les femmes (Tableau 5).

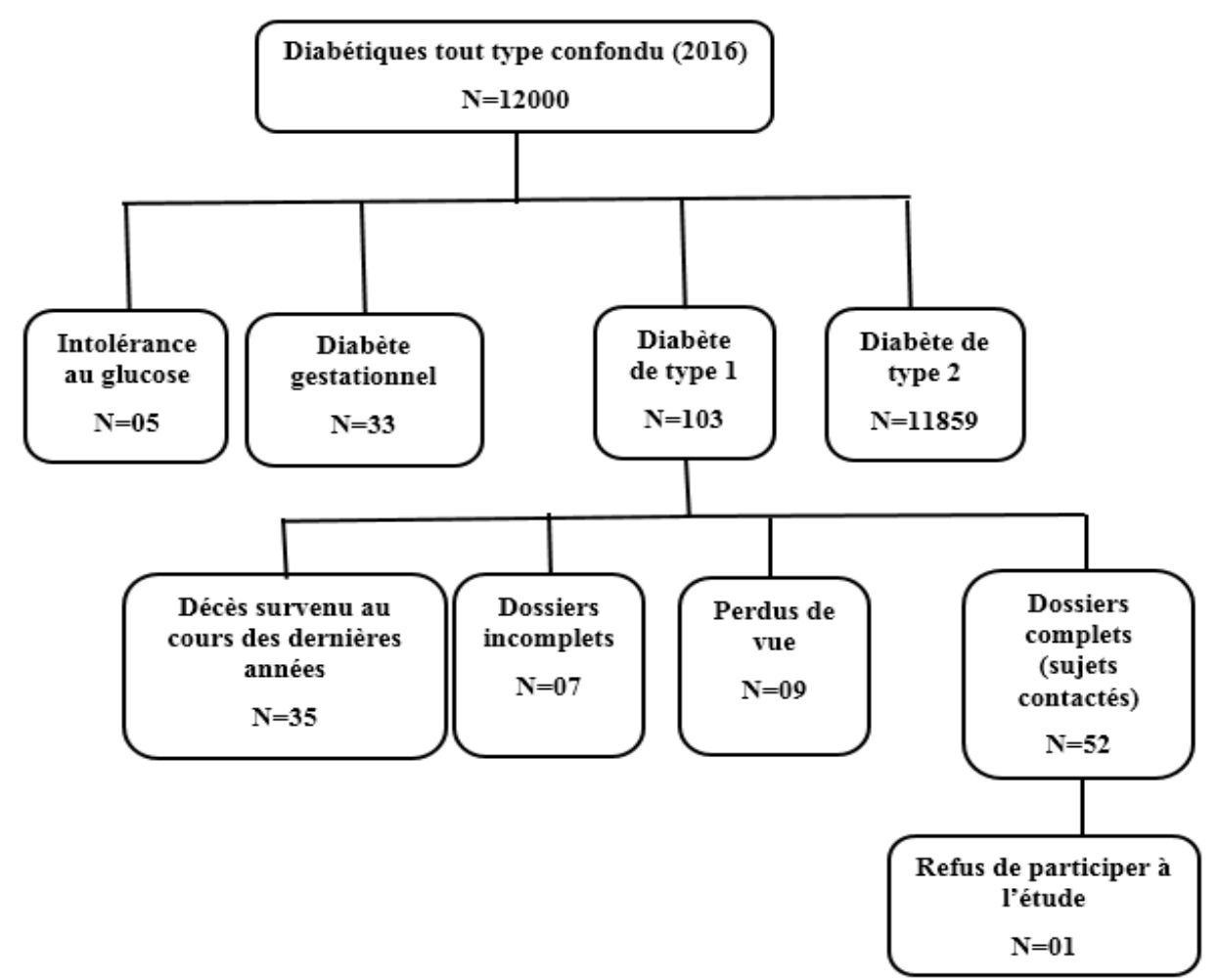

Figure 1 : Flow chart de l'étude des dossiers des diabétiques tout type confondu ayant consulté à la banque d'insuline de Cotonou de janvier 1996 à décembre 2015. 
Tableau 1 : Caractéristiques socio-démographiques des patients diabétiques de type 1 recensés à la Banque d'Insuline de janvier 1996 à décembre 2015.

\begin{tabular}{|c|c|c|c|c|}
\hline Caractéristiques & Nombre & $\begin{array}{l}\text { Fréquence } \\
(\%)\end{array}$ & IC 95\% & $\begin{array}{c}\text { Manque } \\
\text { d'information (\%) }\end{array}$ \\
\hline Sexe & $N=103$ & & & \\
\hline Féminin & 53 & 51,4 & $41,4-61,4$ & 0 \\
\hline Masculin & 50 & 48,6 & $35,7-58,6$ & \\
\hline Age & $\mathrm{N}=103$ & & & \\
\hline$<4$ & 4 & 3,9 & $1,1-9,6$ & \\
\hline$[4-8[$ & 15 & 14,6 & $8,4-22,9$ & \\
\hline$[8-12[$ & 34 & 33,0 & $24,1-43,0$ & 0 \\
\hline$[12-16[$ & 38 & 36,9 & $27,6-47,0$ & \\
\hline$[16-20[$ & 8 & 7,8 & $3,4-14,7$ & \\
\hline$[20-24[$ & 2 & 1,9 & $0,4-8,5$ & \\
\hline [24-28[ & 2 & 1,9 & $0,4-8,5$ & \\
\hline Profession & $\mathrm{N}=98$ & & & \\
\hline Elève & 58 & 59,2 & $48,8-69,0$ & \\
\hline Etudiant & 09 & 9,2 & $4,3-16,7$ & \\
\hline Couturier & 06 & 6,1 & $2,3-12,8$ & \\
\hline Chauffeur & 04 & 4,1 & $1,1-10,1$ & 4,8 \\
\hline Ménagère & 07 & 7,1 & $2,9-14,2$ & \\
\hline Commerçant & 09 & 9,2 & $4,3-16,7$ & \\
\hline Aucune & 05 & 5,1 & $1,7-11,5$ & \\
\hline Ethnie & $\mathrm{N}=92$ & & & \\
\hline Yoruba & 19 & 20,7 & $12,9-30,3$ & \\
\hline Nago & 04 & 4,3 & $1,2-10,7$ & \\
\hline Fon & 38 & 41,3 & $31,1-52,1$ & \\
\hline Goun & 08 & 8,7 & $3,8-16,4$ & 10,7 \\
\hline Mina & 10 & 10,9 & $5,3-19,1$ & \\
\hline Adja & 06 & 6,5 & $2,4-13,6$ & \\
\hline Bariba & 02 & 2,2 & $0,3-7,6$ & \\
\hline Dendi & 04 & 4,3 & $1,2-10,7$ & \\
\hline Kotafan & 01 & 1,1 & $0,1-5,9$ & \\
\hline Antécédents familiaux* & $\mathrm{N}=101$ & & & \\
\hline DT1 & 01 & 1,0 & $0,1-5,4$ & 1,9 \\
\hline DT2 & 41 & 40,6 & $30,9-50,8$ & \\
\hline Néant & 59 & 58,4 & $48,2-68,1$ & \\
\hline
\end{tabular}


Tableau 2 : Caractéristiques sociodémographiques des patients diabétiques de type 1 contactés dans l'étude.

\begin{tabular}{|c|c|c|c|}
\hline Caractéristiques & Nombre & Fréquence (\%) & IC $95 \%$ \\
\hline Sexe & $\mathrm{N}=51$ & & \\
\hline Féminin & 28 & 54,9 & $40,3-68,9$ \\
\hline Masculin & 23 & 45,1 & $31,1-59,7$ \\
\hline Age & $\mathrm{N}=51$ & & \\
\hline$<4$ & 3 & 5,9 & $1,2-16,2$ \\
\hline$[4-8[$ & 5 & 9,8 & $3,3-21,4$ \\
\hline$[8-12[$ & 20 & 39,2 & $25,8-53,9$ \\
\hline$[12-16[$ & 19 & 37,2 & $24,1-51,9$ \\
\hline$[16-20[$ & 03 & 5,9 & $1,2-16,2$ \\
\hline$[20-24[$ & 01 & 2,0 & $0,05-10,4$ \\
\hline Profession & $N=51$ & & \\
\hline Elève & 31 & 60,8 & $46,1-74,1$ \\
\hline Etudiant & 06 & 11,8 & $4,4-23,9$ \\
\hline Couturier & 03 & 5,9 & $1,2-16,2$ \\
\hline Chauffeur & 01 & 2,0 & $0,05-10,4$ \\
\hline Ménagère & 03 & 5,9 & $1,2-16,2$ \\
\hline Commerçant & 04 & 7,8 & $2,2-18,9$ \\
\hline Aucune & 03 & 5,9 & $1,2-16,2$ \\
\hline Ethnie & $N=51$ & & \\
\hline Yoruba & 09 & 17,6 & $8,4-30,9$ \\
\hline Nago & 03 & 5,9 & $1,2-16,2$ \\
\hline Fon & 27 & 52,9 & $38,4-67,1$ \\
\hline Goun & 05 & 9,8 & $3,3-21,4$ \\
\hline Mina & 03 & 5,9 & $1,3-16,2$ \\
\hline Adja & 02 & 3,9 & $0,5-13,4$ \\
\hline Bariba & 01 & 2,0 & $0,05-10,4$ \\
\hline Dendi & 01 & 2,0 & $0,05-10,4$ \\
\hline Antécédents familiaux* & $N=51$ & & \\
\hline DT1 & 01 & 2,0 & $0,05-10,4$ \\
\hline DT2 & 28 & 54,9 & $40,3-68,9$ \\
\hline Néant & 22 & 43,1 & $29,3-57,8$ \\
\hline
\end{tabular}


Tableau 3: Répartition des complications engendrées par le diabète de type 1par type de complication au sein des patients diabétiques de type 1 contactés.

\begin{tabular}{llccc}
\hline Caractéristiques & & Nombre & Fréquence \% & IC 95\% \\
\hline Complications & Hypertension artérielle & $\mathbf{N}=\mathbf{5 1}$ & & \\
d'ordre macro & Oui & 08 & 15,7 & $7,0-28,6$ \\
angiopathies & Non & 43 & 84,3 & $71,4-93,0$ \\
& Dysfonctionnement érectile & $\mathbf{N}=\mathbf{2 3}$ & & \\
& Oui & 15 & 65,2 & $42,7-83,6$ \\
& Non & 08 & 34,8 & $16,4-57,3$ \\
& Irrégularités sévère des menstrues & $\mathbf{N = 2 8}$ & & \\
& Oui & & & \\
& Non & 15 & 53,6 & $33,9-72,1$ \\
& Absence des menstrues & 12 & 42,8 & $24,5-62,9$ \\
& & 01 & 3,6 & $0,1-18,3$ \\
\hline Complications & Acuité visuelle & $\mathbf{N}=\mathbf{5 1}$ & & \\
d'ordre micro & Bonne & 30 & 58,8 & $44,2-72,4$ \\
angiopathies & Mauvaise & 21 & 41,2 & $27,9-55,8$ \\
\hline
\end{tabular}

Tableau 4 : Répartition (\%) des types de diabète sucré à la Banque d'insuline.

\begin{tabular}{lccc}
\hline Types de diabète & Nombre & Fréquence & IC 95\% \\
\hline Diabète de type 2 & 11859 & 98,8 & $98,6-99,0$ \\
Diabète de type 1 & 103 & 0,9 & $0,7-1,0$ \\
PCC* & ---- & --- & ---- \\
Malnutrition & ---- & --- & --- \\
Diabète secondaire & 38 & 0,03 & $0,2-0,4$ \\
\hline \multicolumn{2}{c}{ * pancrétite chronique calcifiante }
\end{tabular}

* pancréatite chronique calcifiante

Tableau 5 : Taux de mortalité au sein de la population diabétique de type 1 recensée (103).

\begin{tabular}{lccc}
\hline & Taux & IC 95\% \\
\hline Mortalité & 34 pour 100 & $24,9-44,0$ \\
Part de mortalité & & \\
- & Hommes & 54,3 pour 100 & $28,8-63,3$ \\
- & Femmes & 45,7 pour 100 & $36,6-71,2$ \\
\hline
\end{tabular}




\section{DISCUSSION}

La prévalence des patients diabétiques a considérablement augmenté dans le monde en raison du style de vie moderne liée à une augmentation de la consommation des aliments à haute teneur en glucides et en graisses (Laleye et al., 2016).

$\mathrm{Au}$ Bénin, parmi les maladies nontransmissibles, le diabète constitue la deuxième maladie la plus fréquente après l'hypertension artérielle. Le diabète de type 1 suscite le plus l'attention des chercheurs en raison des causes très diverses de son déclenchement. Cette maladie a été très peu étudiée au Bénin. L'intérêt de cette étude est de montrer la fréquence du diabète de type 1 parmi les différents types de diabète recensés à la Banque d'Insuline de Cotonou. Au cours de cette étude, le taux moyen de réponses par question pour les variables étudiées est de $96.5 \%$ pour les diabétiques de type 1 recensés. La question ayant obtenu le taux de remplissage le plus bas était celle relative à l'ethnie $(10,7 \%)$. Le biais des données manquantes est commun à toutes les études rétrospectives car leur schéma consiste à sélectionner les cas sur l'apparition

) ont une prédominance masculine, alors que les régions ayant une faible incidence (origine non- européenne) ont une prédominance féminine (Gale et Gillespie, 2001).

De même, il ressort de cette étude que ce sont les enfants diabétiques de la tranche d'âge de [8-16ans [qui sont les plus impliqués dans l'étude $(69,9 \%)$. La population d'étude a concerné plus la couche juvénile, souvent corrélée avec la survenue du diabète de type 1 . Ceci pourrait expliquer la fréquence plus élevée des élèves dans cette étude. Les résultats ont également montré que les patients d'ethnie Fon ont été les plus recensés dans l'étude. Il serait intéressant pour ce constat, d'envisager une étude évaluant une éventuelle implication de de la maladie et toutes les informations ne sont toujours pas enregistrées dans le dossier. Le schéma idéal est l'étude prospective où les sujets sont sélectionnés sur l'exposition. Mais ce schéma nécessite un suivi au cours du temps, parfois long et impose une logistique beaucoup plus lourde. De plus, le recueil méticuleux des données et le rappel téléphonique des parents des enfants ont minimisé le risque de données manquantes, aboutissant à un taux élevé de données accessibles dans cette étude (supérieur à $96 \%$ ).

La répartition des patients DT1 recensés selon le sexe montre que le sexe féminin est le plus représenté (53 soit $51,4 \%$ ) avec un sexratio de 1,06 . Une prédominance du sexe féminin dans le DT1 a été également rapportée dans l'étude de Timóteo et al. (2012) au Portugal. Par contre dans l'étude de JaroszChobot et al. (2011) en Pologne, c'est le sexe masculin qui a été le plus représenté. Des différences d'incidence selon le sexe sont retrouvées cependant dans certaines populations, mais pas toutes (Hanas et al., 2009). Les régions à forte incidence de diabète de type 1 (origine européenne l'alimentation dans le développement de la maladie.

L'appréciation de la part du DT1 dans l'ensemble des cas de diabète de janvier 1996 à décembre 2015 révèle une fréquence du DT1 de $0,9 \%$. La fréquence du DT1 est faible dans toutes les séries en Afrique Subsaharienne. Plusieurs travaux portant sur le diabète sucré en Afrique Subsaharienne rapportent diverses fréquences du diabète de type 1 : Côte d'Ivoire : 10,3\%, Cameroun: 24,7\%, Gabon: 3,8\%, Sénégal: 20\% (Gning et al., 2010). En population générale, des prévalences pour 100.000 personnes de 3,5 ont été retrouvées au Mozambique et 12 en Zambie (Beran et al., 2005). 
Le diabète de type 1 représente un problème majeur de santé publique parce qu'il est associé à long terme à des risques de complications et aux décès prématurés. Au cours de ces vingt dernières années, 35 décès ont été recensés sur les 103 patients diabétiques de type 1 , ce qui représente un taux de mortalité de 34\% avec une part de mortalité de 54,3\% chez les hommes et $45,7 \%$ chez les femmes. Ce taux de mortalité est très élevé et montre la gravité de la maladie et la nécessité d'une attention particulière des autorités sanitaires dans la prise en charge des patients diabétiques de type 1 . Le diabète de type 1 nécessite un engagement permanent du patient pour la gestion du traitement et sa participation active pour les soins quotidiens.

Les personnes qui développent ce type de diabète doivent recevoir des injections quotidiennes d'insuline afin de contrôler le niveau de glycémie. Sans traitement par l'insuline, l'évolution naturelle du DT1 tend vers la mort. Elles peuvent toutefois mener une vie normale et saine grâce à une alimentation saine et des exercices physiques réguliers. Mais, dans un contexte social où le nombre moyen de personnes vivant sous le même toit est de 9 à 10 , avec des ressources financières souvent limitées, le respect d'un régime adéquat est très difficile. Beaucoup de malades, ne pouvant se permettre de consommer seuls à table un régime adapté, sont obligés de prendre les repas familiaux, en tentant de limiter plus ou moins les apports glucidiques. Les deux situations les plus fréquentes sont celle du patient consommant manifestement un excès de glucides, ou celle du patient très mal rassasié après le repas familial.

D'autres facteurs représentent des limites pour une bonne adhésion du patient à son traitement à savoir : la peur des hypoglycémies surtout nocturnes (cas de patient qui a peur d'apporter du glucose et tombe en hypoglycémie), la non-acceptation de la maladie, le refus des contraintes (régime, injections quotidiennes d'insuline), les erreurs techniques (injections trop profondes ou trop superficielles réalisées toujours au même endroit et responsable de lipodystrophies).

Ces différentes situations influenceraient les chances d'obtenir des patients, un bon équilibre glycémique.

Enfin, s'agissant des complications liées au diabète, les perturbations observées au cours de la puberté chez les patients, aussi bien chez le sexe masculin que féminin, pourraient être dues à des modifications du climat hormonal liées à la survenue de la maladie (Zachrisson et Brismar, 2010). En effet, dans le sexe masculin, le dysfonctionnement érectile serait dû à un mauvais contrôle de la glycémie bloquant une étape de la cascade de réactions chimiques mobilisées depuis la stimulation sexuelle jusqu'à l'érection (Galianoa et al., 2010). Schématiquement, l'hyperglycémie s'accompagne d'une diminution de la libération d'oxyde nitrique, une étape pourtant importante à l'augmentation de la concentration en protéines GMPc essentielles à la relaxation des fibres musculaires lisses et donc à l'érection (Musicki et al., 2005; Musicki et Burnett, 2006). Aussi, chez la femme, le diabète de type 1 peut entraîner une perturbation de la sécrétion hypothalamique de la gonadotrophine releasing hormone (GnRH) avec comme résultat une diminution de la sécrétion de l'hormone lutéinisante. Cette dernière a pour rôle de promouvoir la synthèse des androgènes par les cellules thécales de l'ovaire, de déclencher l'ovulation et de maintenir le corps jaune au cours du cycle menstruel (Livshits et Seidman, 2009). Toutefois, un bon contrôle de la maladie peut permettre de réduire considérablement les risques de complications. 


\section{Conclusion}

Ce travail a permis de présenter un état des lieux du diabète de type 1 à la Banque d'insuline de Cotonou. Bien que les présents résultats ne soient pas représentatifs de la population générale, ils renseignent néanmoins sur la maladie dans le système hospitalier béninois. Le fort taux de mortalité obtenu pour cette étude appelle la participation de tous les acteurs : personnels de santé, états, bailleurs de fonds internationaux, qui doivent prendre conscience de la particularité de cette maladie observée le plus souvent chez la couche juvénile.

\section{CONFLIT D'INTERETS}

Les auteurs déclarent qu'il n'y a aucun conflit d'intérêts pour la publication de cet article.

\section{CONTRIBUTIONS AUX AUTEURS}

KAF a effectué la collecte, la saisie et le traitement des données à partir des fiches d'enquêtes. SA est le médecin de l'étude. Il a intervenu pour la sélection des dossiers des patients suivant les critères de diagnostic du DT1. MA a contribué à la rédaction du draft du manuscrit. LB et AL ont supervisé l'étude.

\section{REMERCIEMENTS}

Nos remerciements vont à l'endroit du Ministère de l'Enseignement Supérieur et de la Recherche Scientifique (MESRS) et de l'Institut des Sciences Biomédicales Appliquées (ISBA) du Bénin.

\section{REFERENCES}

American Diabetes Association. 2010. Clinical Practice Recommendations. Diabetes Care, 49(2): S3-3. DOI : https://doi.org/10.2337/dc10-S001.

American Diabetes Association. 2014. Diagnosis and classification of diabetes mellitus. Diabetes Care, 37 Suppl (1) : S81-90. DOI: http://doi.org/10.2337/dc10S062.

Beran D, Yudkin JS, De Courten M. 2005. Access to care for patients with insulinrequiring diabetes in developing countries: case studies of Mozambique and Zambia. Diabetes Care, 28(9): 2136-2140. DOI: https://doi.org/10.2337/diacare.28.9.2136.

Djrolo F, Houinato D, Gbary A, Akoha R, Djigbénoudé O, Sègnon J. 2012. Prevalence of diabetes mellitus in the adult population at Cotonou, Benin. Médecine des Maladies Métaboliques, 6(2): 167-169. DOI : $\quad$ https://doi.org/10.1016/S19572557(12)70386-3.

Gale EAM, Gillespie KM. 2001. Diabetes and gender. Diabetologia, 44(1): 3-15. DOI : http://doi.org/10.1007/s001250051573.

Galianoa M, Pignotbe G, Costad C, Vallanciena G, Virage R. 2010. Dysfonction érectile et cellules endothéliales caverneuses. Progrès Urologique, 20(3): 188-193. DOI: http://doi.org/10.1016/j.purol.2009.07.009.

Gning B, Thiam M, Fall F. 2010. Le diabète sucré en Afrique Subsaharienne: aspects épidémiologiques, difficultés de prise en charge. Médecine Tropicale, 67: 607-611.

Hall V, Thomsen RW, Henriksen O, Lohse N. 2011. Diabetes in sub Saharan Africa 1999-2011: epidemiology and public health implications. A systematic reviex. BMC Public Health., 11: 564. DOI: http://doi.org/10.1186/1471-245811-564.

Hanas R, Donaghue KC, Klingensmith G, Swift PGF. 2009. ISPAD Clinical Practice Consensus Guidelines 2009 Compendium Introduction. Pediatric Diabetes, 10: 1-2. DOI: http://doi.org/10.1111/j.13995448.2009.00577.x.

Jansse OE, Mann K, Erbel R. 2007. Endocrine disorders and the heart. Europe PMC., 
48(3): 246-254. DOI: http://doi.org/ 10.1007/s00108-007-1807-z.

Jarosz-Chobot P, Polanska J, Szadkowska A, Kretowski A, Bandurska-Stankiewicz E, Ciechanowska M, Deja G, Mysliwiec M, Peczynska J, Rutkowska J, SobelMaruniak A, Fichna P, Chobot A, Rewers M. 2011. Rapid increase in the incidence of type 1 diabetes in Polish children from 1989 to 2004, and predictions for 2010 to 2025 . Diabetologia, 54(3): 508-515. DOI: http://doi.org/10.1007/s00125-010-19934.

Koevi KA, Millogo V, Ouedraogo $M$ and Ouedraogo GA. 2014. Diagnostic des causes de complication du diabète et des méthodes de prévention à Bobo-Dioulasso, au Burkina-Faso. Int. J. Biol. Chem. Sci., 8(6): 2709-2720. DOI: http://dx.doi.org/10.4314/ ijbcs.v8i6.30.

Laleye OAF, Ahissou H, Olounlade AP, Azando EVB, Laleye A. 2016. Etude bibliographique de trois plantes antidiabétiques de la flore béninoise: Khaya senegalensis (Desr) A. Juss (Meliaceae), Momordica charantia Linn (Cucurbitaceae) et Moringa oleifera Lam (Moringaceae). Int. J. Biol. Chem. Sci., 9(5): 2682-2700. DOI: http://dx.doi.org/ 10.4314ijbcs.v9i5.38.

Livshits A, Seidman D. 2009. Fertility issues in women with diabetes. Women's Health., 5(6): 701-707.

Mbanya JCN, Motala AA, Sobngwi E, Assah FK, Enorus ST. 2010. Diabetes in subSaharan Africa. The Lancet, 375(9733):
2254-2266. DOI: https://doi.org/10.1016/ S0140-6736(10)60550-8.

Musicki B, Burnett AL. 2006. NOS Function and Dysfunction in the Penis. Experimental Biology and Medecine, 231: 154-165. DOI: https://doi.org/10.1177/ 153537020623100205.

Musicki B, Kramer F, Becker R, Burnett L. 2005. Inactivation of phosphorylated endothelial nitric oxide synthase (Ser1177 ) by O-GlcNAc in diabetes-associated erectile dysfunction. Proceedings of National Academy of Sciences of the United States America., 102(33) : 1187011875. DOI: http://doi.org/10.1073/ pnas.0502488102.

Notkins AL, Lernmark A. 2001. Autoimmune type 1 diabetes: Resolved and unresolved issues. J Clin Invest., 108(9): 1247-1252. DOI: http://doi.org/10.1172/JCI14257.

Organisation Mondiale de la Santé. 2016. Diabète aide-mémoire; $\mathrm{N}^{\circ} 312,4 \mathrm{p}$.

Shaw JE, Sicree RA, Zimmet PZ. 2010. Global estimates of the prevalence of diabetes for 2010 and 2030. Diabetes Res Clin Pract., 87(1): 4-14. DOI: https://doi.org/10.1016/ j.diabres.2009.10.007.

Timóteo C, Castanhinha S, Sampaio L. 2012. Growth and puberty in type 1 diabetes mellitus - experience from a pediatric endocrinology unit. Acta Medica Portuguesa., 25(4): 213-218.

Zachrisson I, Brismar K. 2010. Leptin, Cortisone and Testosterone levels are puberty dependent both in Healthy and Diabetic Boys. Only Leptin is impaired in diabetes, manuscript. 